\title{
Dynamic Nuclear Polarization and Other Magnetic Ideas at EPFL
}

\author{
Aurélien Bornet ${ }^{\star a}$, Jonas Milani ${ }^{a}$, Shutao Wanga ${ }^{a}$ Daniele Mammolia, Roberto Buratto ${ }^{a}$, Nicola \\ Salvi ${ }^{a}$, Takuya F. Segawa ${ }^{a}$, Veronika Vitzthuma ${ }^{a}$ Pascal Miévillea ${ }^{a}$ Srinivas Chinthalapallia, Angel J. \\ Perez-Linde ${ }^{a}$, Diego Carnevale ${ }^{a}$, Sami Jannina, Marc Caporiniab, Simone Ulzega ${ }^{a c}$, Martial Rey ${ }^{a}$, and \\ Geoffrey Bodenhausen adef
}

\begin{abstract}
Although nuclear magnetic resonance (NMR) can provide a wealth of information, it often suffers from a lack of sensitivity. Dynamic Nuclear Polarization (DNP) provides a way to increase the polarization and hence the signal intensities in NMR spectra by transferring the favourable electron spin polarization of paramagnetic centres to the surrounding nuclear spins through appropriate microwave irradiation. In our group at EPFL, two complementary DNP techniques are under investigation: the combination of DNP with magic angle spinning at temperatures near $100 \mathrm{~K}$ ('MAS-DNP'), and the combination of DNP at $1.2 \mathrm{~K}$ with rapid heating followed by the transfer of the sample to a high-resolution magnet ('dissolution DNP'). Recent applications of MAS-DNP to surfaces, as well as new developments of magnetization transfer of ${ }^{1} \mathrm{H}$ to ${ }^{13} \mathrm{C}$ at $1.2 \mathrm{~K}$ prior to dissolution will illustrate the work performed in our group. A second part of the paper will give an overview of some 'nonenhanced' activities of our laboratory in liquid- and solid-state NMR.
\end{abstract}

Keywords: Dissolution DNP · Dynamic nuclear polarization (DNP) · Hyperpolarization · Magic angle spinning (MAS) · MAS-DNP · Nuclear magnetic resonance (NMR)

\section{Introduction}

Our Laboratory of Biomolecular Magnetic Resonance (LRMB) does not merely focus on biomolecules, as its name suggests, but is involved in the development of a broad range of new methods for both solid- and liquid-state NMR. Dynamic Nuclear Polarization (DNP) is perhaps the most spectacular, since it allows one to enhance NMR signals by up to five orders of magnitude, thus enabling studies of systems that were deemed inaccessible until recently. The first part of this paper therefore focuses on some recent

\footnotetext{
${ }^{*}$ Correspondence: A. Bornet ${ }^{\mathrm{a}}$

Tel.: +41216939427

Fax: +41216939435

E-mail: aurelien.bornet@epfl.ch

alnstitut des Sciences et Ingénierie Chimiques,

Ecole Polytechnique Fédérale de Lausanne (EPFL), $\mathrm{CH}-1015$ Lausanne

'Bruker Biospin Corporation, 15 Fortune Drive Manning Park, Billerica, MA 01821-3991, USA 'Bruker BioSpin AG, Industriestrasse 26, $\mathrm{CH}-8117$ Fällanden

dEcole Normale Supérieure, Département de Chimie, 75231 Paris Cedex 05, France.

eUMR 7203, CNRS/UPMC/ENS, Paris, France.

fUniversité de Pierre-et-Marie Curie, Paris, France
}

advances in MAS-DNP and dissolutionDNP. However, some experts do not share the widespread excitement about DNP. In a way, it is no more difficult to flip the switch of a commercial gyrotron than to light a torch in a dark room. It all depends on the contents of the room. When opening Tutankhamen's tomb, it was surely worth having a torch. But a peek into some randomly chosen cave might be disappointing. For this reason, we shall also mention some methods that are not related to DNP in the latter part of this overview.

\section{Dynamic Nuclear Polarization}

Nuclear magnetic resonance often suffers from a lack of sensitivity. Indeed, NMR signal intensities are directly proportional to the nuclear spin polarization. The polarization $P$ of a sample comprising an ensemble of spins $I=1 / 2$ is defined by the difference $\Delta N=N_{\alpha}-N_{\beta}$ of the populations of the states $\alpha$ and $\beta$ with $m=+1 / 2$ and $-1 / 2$, normalized by the total number of spins $N$ $=N_{\alpha}+N_{\beta}$. According to Boltzmann's distribution, the polarization $P$ at thermal equilibrium is given by:

$$
P=\frac{\Delta N}{N}=\tanh \left(\frac{\gamma h B_{0}}{2 k_{B} T}\right)
$$

where $h$ is Plank's constant, $k_{B}$ Boltzmann's constant, and $\gamma$ the gyromagnetic ratio of the spins $I$ placed in a magnetic field $B_{0}$ at a temperature $T$. At room temperature in a magnetic field $B_{0}=3.35 \mathrm{~T}$ (proton Larmor frequency $\left.\omega_{0}=142.7 \mathrm{MHz}\right)$, the proton polarization is only $P\left({ }^{1} \mathrm{H}\right)=1.210^{-5}$. Dynamic Nuclear Polarization (DNP) ${ }^{[1]}$ provides a way of dramatically increasing the polarization and hence the signal intensities in NMR. It consists in transferring the large electron spin polarization of paramagnetic centres to the surrounding nuclear spins through appropriate microwave irradiation. Indeed, due to its higher gyromagnetic ratio, the polarization of electron spins $S=1 / 2$ is $\sim 660$ times larger than that of protons at the same field and temperature. Furthermore, if the temperature is lowered to $T \sim 1 \mathrm{~K}$, the electron spin polarization can approach its maximum $\left(P_{e}\right.$ $\sim 1)$. By comparison, the ${ }^{1} \mathrm{H}$ polarization is only $P\left({ }^{1} \mathrm{H}\right)=3.410^{-3}$ at $T=1 \mathrm{~K}$ and $B_{0}=$ 3.35 T. DNP can lead to enhancements of the nuclear spin polarization by up to five orders of magnitude, although one must carefully define the terms of the comparison. ${ }^{[2]}$

According to Eqn. (1), the temperature has to be as low as possible to obtain the highest polarization. Unfortunately, the resolution of NMR spectra is extremely low near $T \sim 1 \mathrm{~K}$ because of large dipoledipole and other anisotropic interactions. Spinning about the magic angle is exceedingly difficult at very low temperatures, so that little information can be extracted from these spectra. In order to overcome this limitation, several strategies have been developed, and the DNP field is now split 
into different sub-techniques. Two of these are currently investigated in our group: the combination of DNP at $100 \mathrm{~K}$ with magic angle spinning (MAS-DNP) and the combination of DNP at $1.2 \mathrm{~K}$ with rapid heating followed by the transfer of the sample to a high-resolution NMR or MRI system (dissolution-DNP).

Depending on conditions, DNP can occur via different mechanisms. A detailed analysis of these mechanisms is beyond the scope of this paper; very good descriptions can be found in several classic works. ${ }^{[3-6]}$ The first mechanism, that is also easiest to explain, is the solid effect (SE). ${ }^{[7]}$ It is restricted to isolated two-spin systems comprising a single electron spin $S$ coupled by a magnetic dipole-dipole interaction to a single nuclear spin $I$. The transfer of the electron spin polarization to the nucleus is induced by microwave irradiation at frequencies $\omega_{+}=\omega_{e}+\omega_{n}$ or $\omega_{-}=\omega_{e}-\omega_{n}$ (see Fig. 1a), where $\omega_{e}$ and $\omega^{-}$are the electron paramagnetic resonance (EPR) and nuclear magnetic resonance (NMR) frequencies. Such transitions are often called 'forbidden transitions', but are in reality second-order transitions that are partly allowed because of dipolar interactions. Nuclear spin diffusion leads to the propagation of the enhanced polarization through the entire sample volume. SE can occur when the EPR lines are sufficiently narrow, with half-widths $\delta$ that must be smaller than the NMR Larmor frequency $\left(\delta<\omega_{n}\right)$. As it involves a second-order transition, SE requires intense microwave intensities.

The cross effect $(\mathrm{CE})^{[6]}$ involves two electron spins with different EPR frequencies $\omega_{e 1}$ and $\omega_{e 2}$ and one nuclear spin with a Larmor frequency $\omega_{n}$. If the difference in EPR frequencies satisfies the condition $\omega_{e 1}$ $-\omega_{e 2}= \pm \omega_{\eta}$, irradiation at $\omega_{e l}$ produces a local saturation of the first electron that can recover via a spin flip of the second electron at a frequency $\omega_{e 2}$, the difference $\left(\omega_{e 1}\right.$ $-\omega_{e 2}$ ) being compensated by a simultaneous flip of the nuclear spin, thus leading to a net enhancement of its nuclear spin polarization. Biradicals such as TOTAPOL, ${ }^{[9]}$ which can fulfil the condition $\omega_{e 1}-\omega_{e 2}=$ $\pm \omega_{n}$, yield better DNP enhancements than monoradicals like TEMPOL.

When the concentration of the radicals is increased, dipolar interactions between electron spins broaden the homogeneous EPR lines. A third mechanism, known as thermal mixing (TM), can then become dominant.[10,11] TM involves a large number of coupled electrons spins and is best described by a thermodynamic model. The behaviour of electron and nuclear spins can be described in terms of energy reservoirs, each of which is characterized by a spin temperature. ${ }^{[10,12]}$ The electron dipolar reservoir is
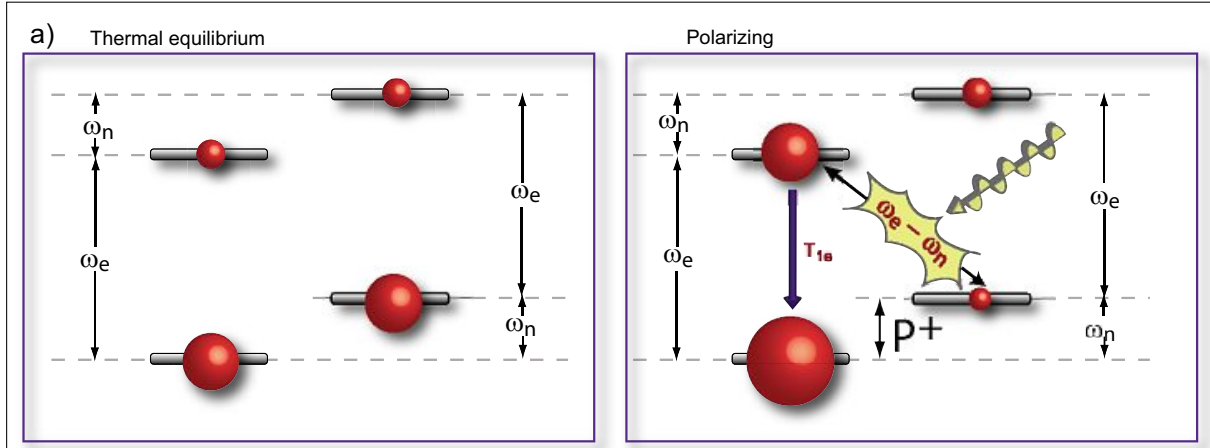

b) Thermal equilibrium
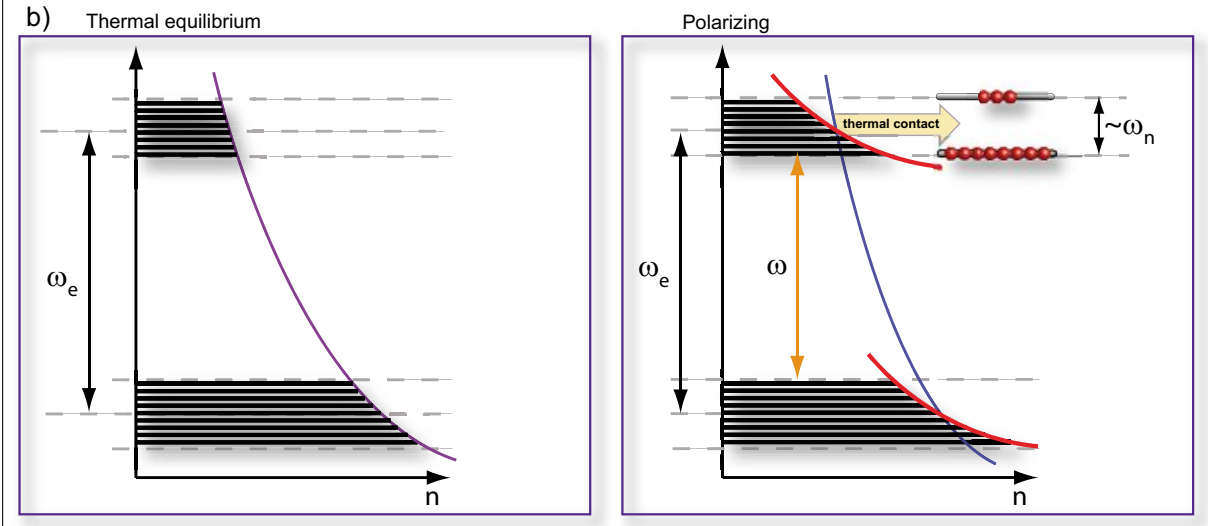

Fig. 1. a) Solid effect in a system comprising a single nuclear spin $I=1 / 2$ and a single electron spin $S=1 / 2$ in the presence of microwave irradiation at $\omega=\omega_{e}-\omega_{n}$. b) Schematic representations of the cooling of the electron dipolar reservoir under microwave irradiation $\omega<\omega_{e}$ and of the thermal contact between the electron dipolar reservoir and the nuclear Zeeman reservoir. (Adapted from Pascal Miéville's PhD thesis. ${ }^{[8]}$ )

cooled down under microwave irradiation and acquires a temperature different from the electron Zeeman reservoir. If the EPR linewidth is larger than the NMR frequency $\left(\delta>\omega_{n}\right)$, the nuclear spin reservoir can then be cooled by thermal contact (Fig. 1b).

\section{Dynamic Nuclear Polarization Combined with Magic Angle Spinning (MAS-DNP)}

MAS-DNP was initially developed by Vriend et al. ${ }^{[13]}$ in 1985 , but only became popular after 1992 in the wake of the remarkable successes of Griffin and co-workers at MIT. [14] Although the expression 'low temperature', which in this context typically refers to the range 90 $<T<110 \mathrm{~K}$, is fully justified for magic angle spinning, it is a bit confusing when comparing with dissolution DNP which is usually carried out at $T=1.2 \mathrm{~K}$. Under favourable conditions, the gain in sensitivity can exceed two orders of magnitude. More typically however, an enhancement factor $\varepsilon_{\text {on/off }}=20$ is regarded as satisfactory, since it leads to a time-saving of $\left(\varepsilon_{\text {on/off }}\right)^{2}=400$, thus allowing to achieve in 24 hours what would normally require more than a year. The overall DNP efficiency $\varepsilon_{\text {tot }}$ depends, among other factors, on the choice and concentration of the (bi)radicals, on the acceleration of the relaxation times that allows one to reduce the recovery delays, on a partial quenching of the signal intensity, on the temperature, and on the static magnetic field.

Performing solid-state DNP experiments at high magnetic fields requires several costly accessories in addition to a normal MAS setup. Currently, only gyrotrons can generate microwave fields that are sufficiently intense to saturate the EPR transitions, i.e., about $5 \mathrm{~W}$ in continuous mode. For NMR spectrometers operating at proton frequencies of 400,600 or $800 \mathrm{MHz}$ $(4.9,14$ or $9.8 \mathrm{~T})$, the microwave frequencies must be $263.7,395.6$, and $527.5 \mathrm{GHz}$, respectively. A sophisticated 'corrugated' low-loss transmission line guides the microwaves from the gyrotron to the sample that is spinning at rates on the order of $10<$ $v_{\text {rot }}<15 \mathrm{kHz}$ in a specially designed MAS probe. A suitable set of heat exchangers generates three separately controlled jets of nitrogen to cool the sample, support the bearing, and drive the spinning of the rotor. In our Bruker prototype, this required no less than 800 liters of liquid nitrogen per day, but more efficient heat exchangers are under development. Fig. 2 shows a schematic layout and a picture of our laboratory at EPFL. The main components and their characteristics have been described in detail by Rosay et al. ${ }^{[16]}$ 


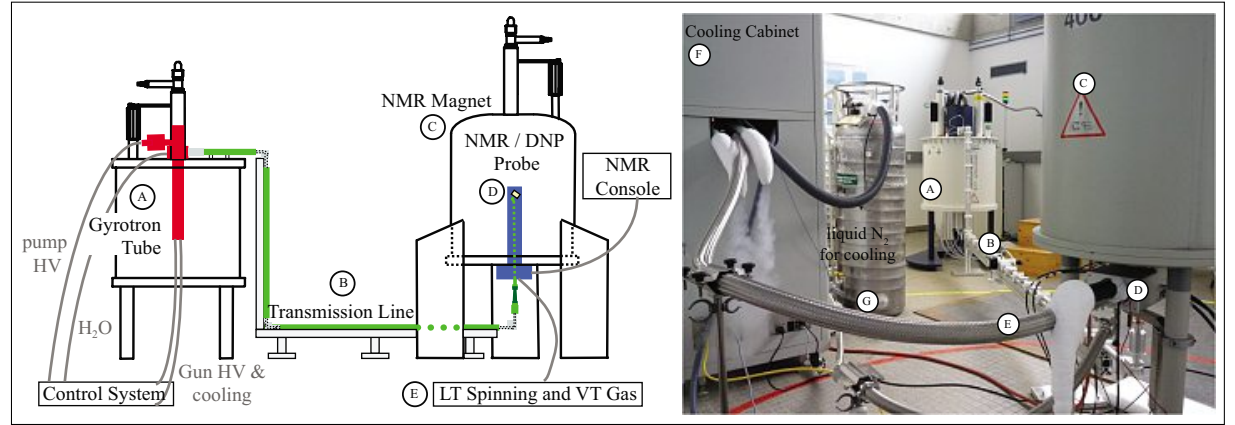

Fig. 2. Schematic and actual DNP set-up at EPFL. Microwaves with a wave-length of $1.14 \mathrm{~mm}$ (ca. $5 \mathrm{~W}$ at $263.7 \mathrm{GHz}$ ) are generated by a gyrotron tube inserted into a 9.4 T magnet (A) and transmitted via the transmission line (B) to the MAS probe (D) in the NMR magnet (C), in our case an aging wide-bore 9.4 T magnet for proton NMR at $400 \mathrm{MHz}$ that can be used in triple-channel mode $\left({ }^{1} \mathrm{H},{ }^{13} \mathrm{C},{ }^{14} \mathrm{~N}\right.$ or $\left.{ }^{1} \mathrm{H},{ }^{13} \mathrm{C},{ }^{15} \mathrm{~N}\right)$ or in double-channel mode (typically ${ }^{1} \mathrm{H}$ plus ${ }^{13} \mathrm{C}$, ${ }^{29} \mathrm{Si}$, or ${ }^{27} \mathrm{Al}$ ). The zirconia or sapphire rotors with an outer diameter of $3.2 \mathrm{~mm}$ are spun up to $v_{\text {rot }}=16 \mathrm{kHz}$ at temperatures near $T=100 \mathrm{~K}$ using three nitrogen gas flows for cooling, bearing and spinning (E) that are regulated by a heat exchanger $(F)$ driven with gaseous and liquid nitrogen $(G)$. (Adapted from Veronika Vitzthum's EPFL thesis. $\left.{ }^{[15]}\right)$

The sample preparation is often the key to achieve the best signal-to-noise ratio. The difference between standard samples for solid-state NMR and DNP samples is the requirement of homogeneously distributed nitroxide radicals like TEMPOL or biradicals like TOTAPOL. Their concentration must be optimized to maximize DNP. The radicals are usually dissolved in a partly deuterated glass-forming solvent, unless they can be attached covalently to the molecules or matrices of interest.

Recently we discovered that MASDNP has a tremendous potential for the characterization of functionalized surfaces of hybrid materials. ${ }^{[17]}$ The initial samples were provided by the group of Christophe Copéret, who moved from Lyon to ETH Zürich in the course of our collaboration. Experiments were carried out with the help of colleagues at the Centre de RMN à Très Hauts Champs in Lyon, in particular Anne Lesage and Moreno Lelli, under the direction of Lyndon Emsley. Normally, the appalling sensitivity of NMR does not allow one to consider surface studies. Even in samples with high surface-to-bulk ratios, as in porous nanoparticles, the concentration of grafted species is typically below 1 $\mathrm{mmol} / \mathrm{g}$ for ${ }^{1} \mathrm{H}$ and $0.01 \mathrm{mmol} / \mathrm{g}$ for ${ }^{13} \mathrm{C}$ in natural abundance, requiring many hours or even days to accumulate simple one-dimensional spectra with reasonable signalto-noise ratios. This forbids the acquisition of multidimensional correlation spectra, thus severely limiting the power of solidstate NMR techniques. When boosted by DNP, solid-state NMR can not only reveal ${ }^{13} \mathrm{C}$ signals of organic molecules grafted on surfaces, but also correlations between ${ }^{1} \mathrm{H}$ signals of such molecules and ${ }^{29} \mathrm{Si}$ or ${ }^{27} \mathrm{Al}$ sites on these surfaces. A detailed characterization of the structure of surface species is likely soon to contribute to a better understanding of heterogeneous catalysis.
This may well lead to improved properties of heterogeneous catalysts through the study of structure-activity relationships, in analogy to similar work on biomolecules. We began by investigating DNP-enhanced ${ }^{13} \mathrm{C}$ signals of nanoporous silica materials functionalized with phenol and imidazolium groups. ${ }^{[17,18]}$ Next, we considered DNP-enhanced ${ }^{29} \mathrm{Si}$ signals of hybrid silica materials where organic groups are covalently bound to a silica matrix. ${ }^{[19]}$ Finally, we observed DNP-enhanced signals of quadrupolar ${ }^{27} \mathrm{Al}$ nuclei $(I=5 / 2)$ near the surface of $\gamma$-alumina nanoparticles. ${ }^{[20]}$ As the samples are by nature heterogeneous and usually do not contain any solvents, DNP sample preparation had to be adapted to surface species. In contrast to earlier MAS-DNP experiments, where the analytes are suspended in partly deuterated glycerol/water mixtures, we used 'incipient wetness impregnation' [21] with aqueous $\mathrm{H}_{2} \mathrm{O} / \mathrm{D}_{2} \mathrm{O}$ solutions of TOTAPOL without glycerol. To obtain structural information about the surfaces of these materials, one needs a method to discriminate between signals from the surface and signals from the bulk. This can be achieved by transferring the magnetization via cross polarization (CP) from the DNP-enhanced ${ }^{1} \mathrm{H}$ to ${ }^{13} \mathrm{C},{ }^{29} \mathrm{Si}$ or ${ }^{27} \mathrm{Al}$ nuclei on the surface.

Fig. 3 shows typical DNP enhancements for ${ }^{13} \mathrm{C}$ nuclei of phenol groups grafted on porous silica. With $25 \mathrm{mM}$ of TOTAPOL, we observed a direct enhancement $\varepsilon_{\mathrm{H}}=25$ of the proton signals of the frozen solvent in the pores. The ${ }^{13} \mathrm{C}$ enhancement $\varepsilon_{\mathrm{C}} \geq 56$ demonstrates that the polarization can be transferred efficiently from ${ }^{1} \mathrm{H}$ to ${ }^{13} \mathrm{C}$ of the surface via $\mathrm{CP}$. The spectrum was obtained in less than $35 \mathrm{~min}$. Due to the presence of paramagnetic species, the relaxation times $T_{1}$ of the protons are shortened. This makes it possible to use short recovery delays, and

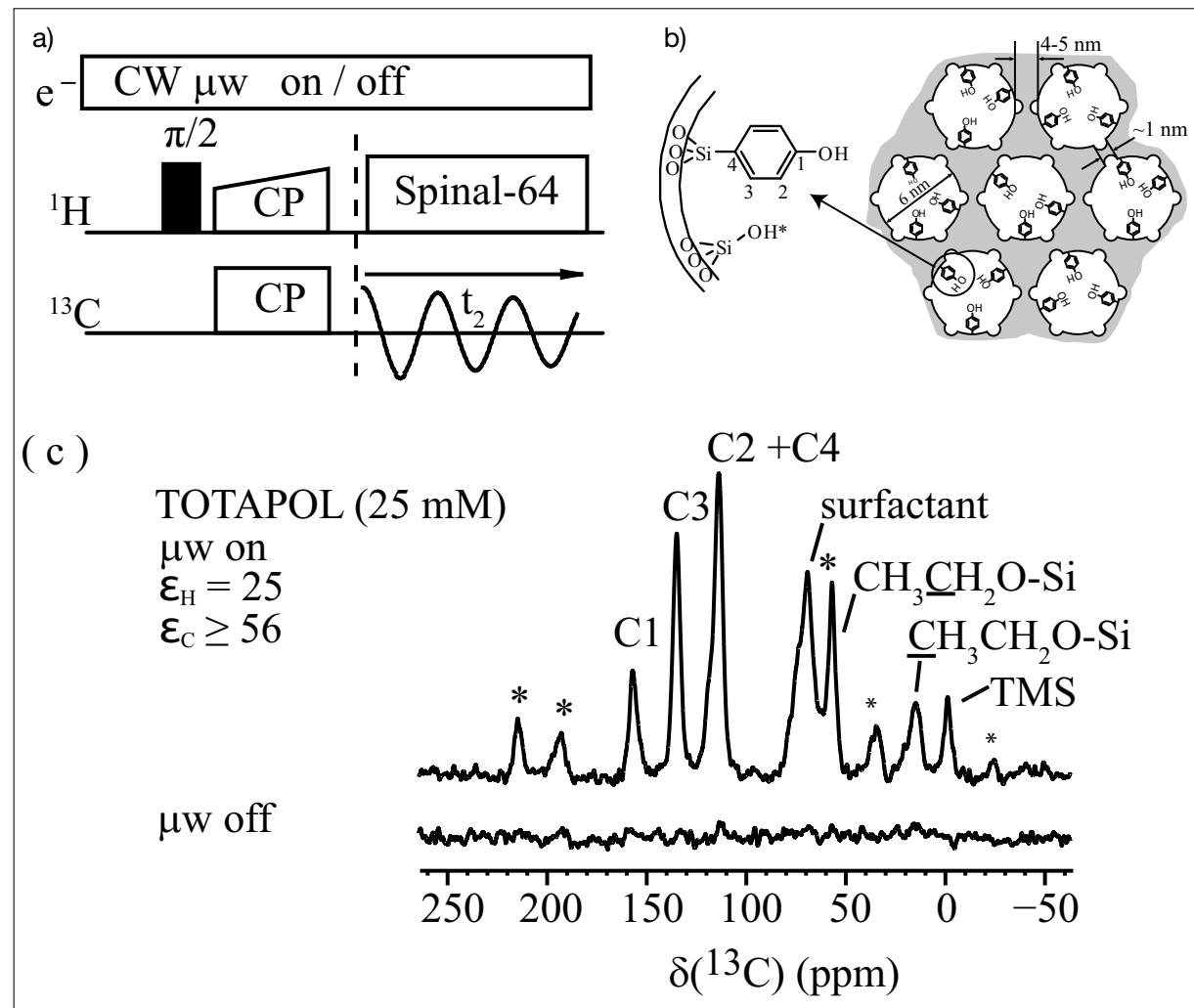

Fig. 3. (a) Pulse sequence used for one-dimensional MAS-DNP with cross polarization (CP) from the DNP-enhanced ${ }^{1} \mathrm{H}$ to ${ }^{13} \mathrm{C},{ }^{29} \mathrm{Si}$ or ${ }^{27} \mathrm{Al}$. (b) Schematic representation of phenol group grafted on porous silica. (c) Carbon-13 CP-MAS-DNP spectra of phenol groups grafted on porous silica with microwaves off or on. (Adapted from Lesage et al.., ${ }^{[18]}$ with permission of the American Chemical Society.) 
thus accelerate the accumulation of many transients. Without DNP, it would take at least 70 days to obtain a carbon-13 spectrum with the same signal-to-noise ratio! The acquisition of 2D spectra, which is inconceivable without polarization enhancement, opens the way to a detailed characterization of the materials.

DNP-enhanced ${ }^{29} \mathrm{Si}$ signals of hybrid materials (typically $\varepsilon_{\mathrm{Si}}=20$ ) allow one to differentiate between different preparation methods (e.g., sol-gel preparation vs. post-grafting.) The spectra show different patterns as a function of the bonding scheme and the geometry on the surface. Here again, DNP allows one to acquire enhanced multi-dimensional spectra.

Finally, the combination of DNP to enhance the proton magnetization with cross polarization (CP) from protons to ${ }^{27} \mathrm{Al}$ allowed us to determine the symmetry of ${ }^{27} \mathrm{Al}$ sites on the surface of $\gamma$-alumina nanoparticles. Triple quantum experiments (TQ-MAS) were used to improve the resolution. This was achieved by first transferring the magnetization from ${ }^{1} \mathrm{H}$ to ${ }^{27} \mathrm{Al}$ single-quantum coherence (SQC) via $\mathrm{CP}$, then converting this SQC to longitudinal magnetization of ${ }^{27} \mathrm{Al}$, and finally by conversion to TQC. ${ }^{[22]}$ Fig. 4b shows a DNPenhanced MQ-MAS spectrum acquired in $\sim 50 \mathrm{~h}$ with the pulse sequence of Fig. 4a. Octahedral $\mathrm{Al}(\mathrm{VI})$ and tetrahedral $\mathrm{Al}(\mathrm{IV})$ aluminium sites can be clearly identified. Surface ${ }^{27} \mathrm{Al}$ signals were enhanced by DNP by a factor $\varepsilon_{\mathrm{Al}}=20$, corresponding to a time saving factor of 400 .

\section{Dissolution DNP}

Another application of DNP has been developed since 2003 by ArdenkjaerLarsen et al. ${ }^{[2]}$ This has come to be known as 'dissolution-DNP'. It consist in rapidly dissolving a frozen hyperpolarized sample (typically at about $1.2 \mathrm{~K}$ ) by a burst of hot $\mathrm{D}_{2} \mathrm{O}$ vapour to obtain a dilute solution at room temperature, while preserving the enhanced nuclear spin polarization. This hyperpolarized solution can then be transferred to a high-resolution NMR spectrometer or MRI scanner. As the main DNP mechanism is usually thermal mixing (TM), the EPR linewidth of the free radical should be larger than the NMR frequency of the nuclei of interest $\left(\delta>\omega_{n}\right)$. This condition can be readily fulfilled by trityl for carbon-13 since $\delta_{\text {trityl }}>\omega_{\mathrm{n}}\left({ }^{13} \mathrm{C}\right)$. At the same time, $\delta_{\text {trityl }}<\omega_{\mathrm{n}}\left({ }^{1} \mathrm{H}\right)$, so that the protons are hardly polarized, thus avoiding a leakage of polarization through the proton bath. Trityl is also useful for direct enhancement of other low-gamma nuclei. To enhance protons however, the common (and inexpensive) radical TEMPO has to be preferred, since its broader EPR linewidth

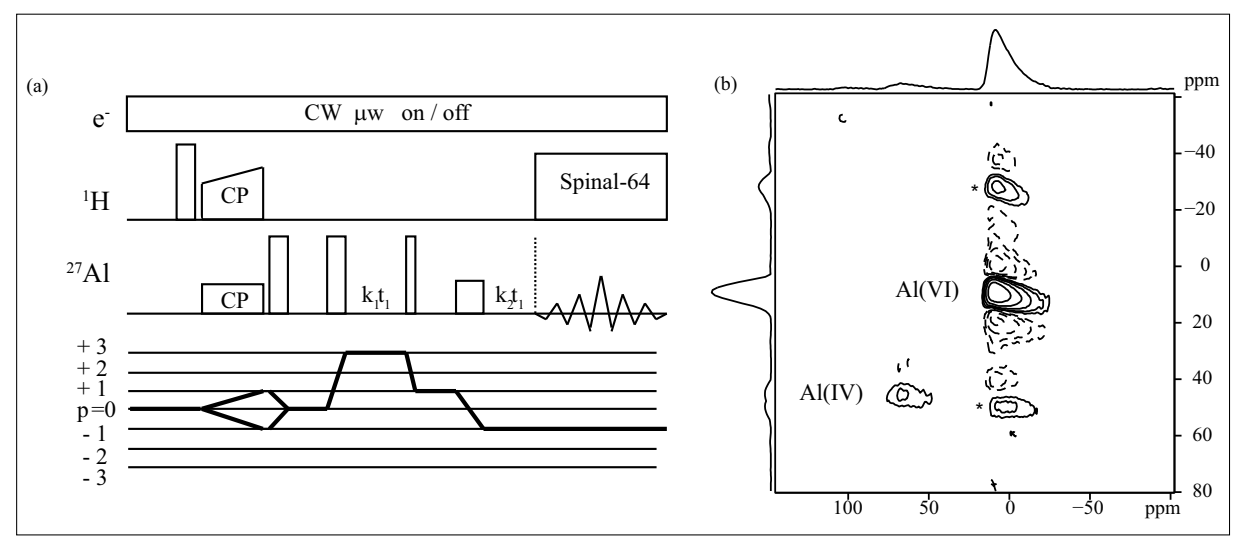

Fig. 4. (a) Pulse sequence used for DNP-enhanced cross-polarization combined with shiftedecho triple-quantum magic-angle spinning experiments (CP-TQ-MAS) with coherence transfer pathways for ${ }^{27} \mathrm{Al}$. (b) DNP-enhanced CP-TQ-MAS spectrum of ${ }^{27} \mathrm{Al}$ in $\gamma$-alumina recorded with the sequence shown in (a) acquired in ca. $50 \mathrm{~h}$. Octahedral $\mathrm{Al}(\mathrm{VI})$ and tetrahedral $\mathrm{Al}(\mathrm{IV})$ aluminium sites can clearly be identified, but no penta-coordinated sites can be seen. (From Vitzthum et al., ${ }^{[20]}$ reproduced with permission of the Royal Society of Chemistry)

fulfils the condition $\delta_{\text {TEMPO }}>\omega_{n}\left({ }^{1} \mathrm{H}\right)$. At EPFL, dissolution-DNP is performed with home-built polarizers. ${ }^{[23]}$ In our laboratory, we use a continuous-flow ${ }^{4} \mathrm{He}$ cryostat operating at $T=1.2 \mathrm{~K}$ which can be inserted into wide-bore cryomagnets with $B_{0}=3.35$ or 6.7 T. Microwave irradiation at $f_{u w}=93.5$ or $187 \mathrm{GHz}$ generated by an ELVA microwave source provides a maximum power $P=400$ or $100 \mathrm{~mW}$. The apparatus at EPFL was developed in close collaboration with the Paul Scherrer Institute (with P. Hautle and J. A. Konter.) A modified probe can be used to generate large quantities of hyperpolarized gaseous samples, in particular hyperpolarized ${ }^{129} \mathrm{Xe}$, without requiring any optical equipment.[24]

Dissolution-DNP was originally developed in view of in vivo MRI applications. It has been shown that the enzymatic inter-conversion of pyruvate and lactate is significantly faster in cancer cells than in healthy tissue. ${ }^{[25]}$ This opens new perspectives for cancer diagnosis, in particular for prostate cancer. To maximize the signal-tonoise of pyruvate after injection into living animals and humans, it is preferable to use DNP-enhanced isotopically enriched ${ }^{13} \mathrm{C}$ pyruvate, which has a slow $T_{1}$ relaxation, and features a response that is not hidden by background signals.

We believe that, no matter how exciting the medical implications may be, we shall see many interesting in vitro applications of dissolution DNP. Indeed dissolution DNP has been used to boost signals of lithium- $6^{[26]}$ and we have explored some exotic uses of yttrium-89.[27] In the latter case, we could determine the slow rate of the formation of a complex between yttrium $\mathrm{Y}^{3+}$ ions and DOTAM as ligand by using a series of $5^{\circ}$ radio frequency pulses extending over 20 minutes in a single DNP experiment.

\section{Long-lived States to Preserve High Polarization}

The life-time of the DNP-enhanced polarization is normally limited by the longitudinal (spin-lattice) relaxation time $T_{1}$, which tends to be short when the sample is travelling through the so-called 'death valley' on its voyage between the polarizer and the NMR or MRI magnet, through an area where the field can drop as low as the earth's field, i.e., about $35 \mu \mathrm{T}$. This problem can be avoided in part by 'sandwiching' the polyethelene tube that carries the solution (typically a $2.5 \mathrm{~mm}$ outer diameter tube) between rows of small permanent magnets. If one uses alloys of neodymium, iron and boron, one can maintain a field close to $1 \mathrm{~T}$ during the entire voyage. In addition, provided the sample contains scalar-coupled pairs of spins, the polarization can be preserved by a temporary conversion into long-lived states.[28,29]

\section{Vitamin C Extends Life Expectancy!}

The shortening of the longitudinal relaxation time $T_{1}$ in the weak field between the two magnets becomes particularly severe when the solution contains radicals. ${ }^{[30]}$ The resulting losses in polarization can be avoided by 'scavenging' the radicals by a chemical reduction agent. Thus the popular radical TEMPO can be readily reduced by ascorbic acid (vitamin C). In practice, the polarizer is loaded with frozen droplets ('beads') of the analyte dissolved in a glass-forming solvent containing $\mathrm{ca} .30$ mM TEMPO, which are mixed with frozen beads of a $3 \mathrm{M}$ aqueous solution of ascorbic acid. Upon dissolution with hot water, both types of beads rapidly melt, and the reduction occurs during the voyage from the polarizer to the NMR or MRI magnet. ${ }^{[31]}$ 


\section{The Race for the Highest Possible Polarization}

In contrast to MAS-DNP, dissolutionDNP must be carried out as a 'one shot' experiment. Therefore it is of paramount importance to achieve the highest possible polarization in the shortest possible time. With trityl and similar radicals that have narrow EPR lines, a polarization as high as $P\left({ }^{13} \mathrm{C}\right)=36 \%$ can be reached at $3.35 \mathrm{~T}$ and $0.8 \mathrm{~K} .{ }^{[32]}$ However, in a moderate field $B_{0}=3.35 \mathrm{~T}$, the build-up time constants are on the order of $35 \mathrm{~min}$ at $T=1.2 \mathrm{~K}$, so that injections of fully polarized samples can only be done once every $100 \mathrm{~min}$ or so. Hence, it is not the installation of the patient in the MRI scanner that would be the time-limiting factor in clinical applications, but the DNP build-up time. One way to overcome this issue is to develop multiple injectors. ${ }^{[32,33]}$ Another possibility to speed up the DNP process is to take advantage of the faster build-up of ${ }^{1} \mathrm{H}$ polarization, and to use cross polarization to transfer the magnetization from protons to carbon-13 in the polariser before dissolution. As we have seen above, TEMPO turns out to be a good option for protons. At a high field $B_{0}=6.7 \mathrm{~T}$ and $T=1.2 \mathrm{~K}$, a proton polarization $P\left({ }^{1} \mathrm{H}\right)=94 \%$ can be readily achieved with $50 \mathrm{mM}$ TEMPO, and preliminary attempts of cross-polarization have yielded a promising carbon polarization $P\left({ }^{13} \mathrm{C}\right)=71 \%$. Even at a moderate field $B_{0}=3.35 \mathrm{~T}$ and $T=1.2 \mathrm{~K}$, a proton polarization $P\left({ }^{1} \mathrm{H}\right)=40 \%$ can be achieved with $30 \mathrm{mM}$ TEMPO, and cross-polarization yields a carbon polarization $P\left({ }^{13} \mathrm{C}\right)=25 \%$. The build-up is much faster for protons than for carbon-13. At a high field $B_{0}=$ $6.7 \mathrm{~T}$ and $T=1.2 \mathrm{~K}, \tau_{\text {DNP }}\left({ }^{1} \mathrm{H}\right)=150 \mathrm{~s}$ and $\tau_{\mathrm{DNP}}\left({ }^{13} \mathrm{C}\right)=1980 \mathrm{~s}$. In a moderate field $B_{0}$ $=3.35 \mathrm{~T}$ and $T=1.2 \mathrm{~K}, \tau_{\mathrm{DNP}}\left({ }^{1} \mathrm{H}\right)=81 \mathrm{~s}$ and $\tau_{\text {DNP }}\left({ }^{13} \mathrm{C}\right)=890 \mathrm{~s}$.

Initially explored by Angel Joaquin Perez Linde and Walter Kockenberger in Nottingham, ${ }^{[34]}$ the use of cross polarization $(\mathrm{CP})$ to transfer ${ }^{1} \mathrm{H}$ polarization to ${ }^{13} \mathrm{C}$ prior to dissolution was further developed at EPFL. ${ }^{[35,36]} \mathrm{CP}$ was carried out on a static sample at $1.2 \mathrm{~K}$ in the DNP polarizer with a custom-built doubly tuned NMR probe developed in collaboration with Roberto Melzi (Bruker BioSpin). The two $r f$ fields, each with an amplitude of $55 \mathrm{kHz}$, fulfill the Hartmann-Hahn condition without arcing in the low-pressure helium bath. As the ${ }^{1} \mathrm{H}$ spectra have linewidths of about $50 \mathrm{kHz}$, this is barely sufficient to provide efficient CP. Fig. 5 shows the DNP build-up curve of the ${ }^{13} \mathrm{C}$ polarization in $1-{ }^{13} \mathrm{C}$ labelled acetate with and without $\mathrm{CP}$. In the former case, $\mathrm{CP}$ contacts were repeated every 2 min. At $B_{0}=3.35 \mathrm{~T}$ and $T=1.2 \mathrm{~K}$, a polarization $P\left({ }^{13} \mathrm{C}\right)=23 \%$ was achieved with $30 \mathrm{mM}$ TEMPO with a build-up time $\tau_{\mathrm{DNP}}$
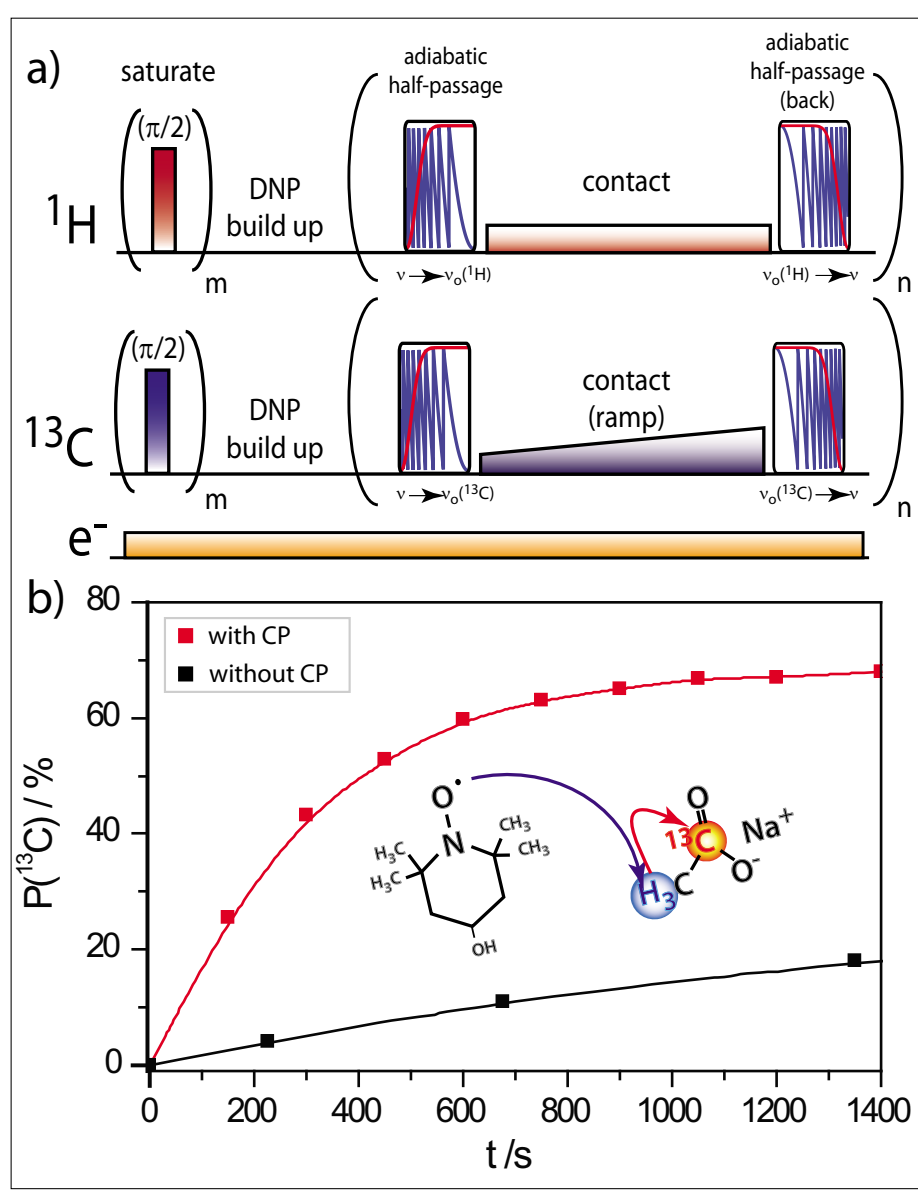

Fig. 5. a) Adiabatic half-passage crosspolarization (CP) used to transfer DNP enhanced polarization from ${ }^{1} \mathrm{H}$ to ${ }^{13} \mathrm{C}$. The frequency-swept pulses applied to the ${ }^{1} \mathrm{H}$ and ${ }^{13} \mathrm{C}$ spins before and after the contact serve to flip their magnetization vectors along the spin-locking field and back to the $z$-axes in view of multiple contacts and subsequent dissolution. b) DNP build-up of the ${ }^{13} \mathrm{C}$ polarization measured with (red) and without cross-polarization (CP) (black) with $B_{0}=$ 6.7 $\mathrm{T}$ and $T=1.2 \mathrm{~K}$, monitored by applying $5^{\circ}$ pulses.

$=170 \mathrm{~s}$. Thus, the build-up is accelerated by a factor 5.2 . At $B_{0}=6.7 \mathrm{~T}$ and $T=1.2 \mathrm{~K}$, a polarization $P\left({ }^{13} \mathrm{C}\right)=71 \%$ was achieved with $50 \mathrm{mM}$ TEMPO with a build-up time $\tau_{\mathrm{DNP}}=488 \mathrm{s.}{ }^{[37]}$ In this case, the build-up is accelerated by a factor 4 . This means that a good ${ }^{13} \mathrm{C}$ polarization can be achieved in less than 15 min. With CP combined with TEMPO, the polarization build-up step is no longer the time-limiting factor of dissolution-DNP experiments. We believe that further improvements in hardware, sample preparation and pulse sequence design may lead to higher enhancements.

\section{Some other Magnetic Ideas}

The second part of this paper gives an overview of some other activities in liquidand solid-state NMR that are not related to DNP.

For many customers of routine NMR spectrometers, decoupling is a fundamental tool to simplify spectra. Whereas heteronuclear decoupling has become a routine operation, homonuclear decoupling remains a challenge. ${ }^{[38,39]}$ We developed ${ }^{40]}$ a new one-dimensional (1D) technique where a comb of very short radio-frequency ( $r f)$ pulses with moderate amplitudes are applied during signal acquisition, in the intervals ('dwell times') between the observation of data points. This approach allows one to decouple simultaneously multiple resonances and allows one to determine the remaining coupling constants.

A more sophisticated approach exploits spin echoes, which are normally modulated by homonuclear scalar couplings. The suppression of echo modulations allows one to measure the transverse relaxation times $T_{2}$ of protons in coupled spin systems. These methods can also be used for biomolecules that are isotopically enriched in ${ }^{13} \mathrm{C}$ or ${ }^{15} \mathrm{~N}$, where echo decays tend to be masked by $J$-modulations.

A third field of research concerns novel tools to study chemical exchange induced by slow motions, particularly in proteins, by observing the lifetimes of heteronuclear multiple-quantum (MQ) coherences in ${ }^{1} \mathrm{H}-{ }^{15} \mathrm{~N}$ spin pairs. ${ }^{[41-43]}$ Observing MQ relaxation rather than single-quantum (SQ) can provide information about two spins that are simultaneously affected by chemical exchange. This technique allows one to stretch the accessible timescales from milliseconds down to microseconds where many conformational motions occur that are relevant to biological process.

A further field of research in liquids is concerned with long-lived states (LLS) ${ }^{[44]}$ and long-lived coherences (LLCs). ${ }^{[45,46]}$ In a system of two magnetically equivalent spins with $I=1 / 2$, the population difference between the anti-symmetric singlet state and the three symmetric triplet states has a 
lifetime $T_{\text {LLS }}$ that can be much longer than the longitudinal ('spin-lattice') relaxation time $T_{1}$. The dipole-dipole interaction, which is usually the main relaxation mechanism in liquid state NMR, cannot interconvert populations between singlet and triplet states. In a recent study, we used this property to enhance the contrast in protein-ligand screening by NMR.[47] Using LLS, we could decrease the minimum protein-to-ligand ratio by at least a factor 25 compared to established NMR techniques, like the $T_{1 \rho}$ method. This makes it possible either to save on costly protein, or to increase the concentration and hence the signal intensity of the observed ligand, which allows one to save experimental time.

Long-lived coherences (LLCs) have a similar relationship to LLS as transverse magnetization $M_{x}$ to longitudinal magnetization $M_{z}$. LLCs decay with a time constant $T_{\text {LLC }}$ that can be much longer than $T_{2}$, but, in contrast to LLS, they oscillate because of scalar couplings. A Fourier transform of their oscillatory decays gives $J$ spectra with very narrow line-widths $\Delta v_{\text {LLC }}=1$ / $\left(\pi T_{\text {LLC }}\right)$. We recently developed a method to acquire LLC spectra 'on-the-fly', in one single transient. ${ }^{[48]}$ It is possible to obtain $J$ spectra with line-widths as small as 14 $\mathrm{mHz}$ in less than $100 \mathrm{~s}$. Moreover, since LLCs are insensitive to the inhomogeneity of the static field, we developed a 2D sequence that correlates zero-quantum coherences (ZQCs) with frequencies given by chemical shift differences $\Delta \mathrm{v}_{I S}$ on the one hand, with scalar couplings $J_{I S}$ on the other. We were able to determine $\Delta \mathrm{v}_{I S}$ and $J_{I S}$ with line-widths of 1 and $0.1 \mathrm{~Hz}$ respectively, even in a very inhomogeneous field where normal proton spectra show linewidths of about $5000 \mathrm{~Hz} .{ }^{[49]}$

In solid-state NMR, much of our attention has been focused on the indirect detection of ${ }^{14} \mathrm{~N}$. Nitrogen-14 has a favourable natural abundance $(99.6 \%)$ and a gyromagnetic ratio that is not much worse than nitrogen-15, since $\gamma\left({ }^{14} \mathrm{~N}\right) / \gamma\left({ }^{15} \mathrm{~N}\right) \sim 0.70$ and $\gamma\left({ }^{14} \mathrm{~N}\right) / \gamma\left({ }^{1} \mathrm{H}\right) \sim 0.07$. Unfortunately, ${ }^{14} \mathrm{~N}$ has a spin $I=1$ like ${ }^{2} \mathrm{H}$, and a quadrupolar splitting that can be larger than $3 \mathrm{MHz}$ in rigid molecules. Even when spinning rapidly, ${ }^{14} \mathrm{~N}$ spectra often feature dozens of spinning sidebands, ${ }^{[50]}$ rendering their excitation and detection very challenging. We therefore developed methods that combine MAS with indirect detection of ${ }^{14} \mathrm{~N}$ via neighbouring spy nuclei with a more favourable spin $S=1 / 2$ such as ${ }^{1} \mathrm{H}$ or ${ }^{13} \mathrm{C}$. ${ }^{[51-54]}$ These methods are closely related to the well-known heteronuclear single- and multiple-quantum correlation experiments (HSQC and HMQC) in liquids. Indirect detection of ${ }^{14} \mathrm{~N}$ in solids was used to study local dynamics in peptides and revealed motions on time-scales near $10^{-7} \mathrm{~s}$ that had never been observed before by NMR. ${ }^{[55]}$
The overall efficiency of indirect detection of ${ }^{14} \mathrm{~N}$ can be greatly improved by replacing the rectangular excitation and reconversion pulses by trains of short rotor-synchronized pulses based on the wellknown DANTE scheme. ${ }^{[56]}$ This allowed us to excite very large families of spinning sidebands in a uniform manner. ${ }^{[57]}$

Finally, new composite pulses have been developed for the excitation of the central transitions (CT) of half-integer quadrupolar nuclei like ${ }^{23} \mathrm{Na}(I=3 / 2),{ }^{27} \mathrm{Al}$ $(I=5 / 2)$ and ${ }^{45} \mathrm{Sc}(I=7 / 2)$. ${ }^{[58]}$ The CT signals of these and many other ubiquitous nuclei tend to be broadened by anisotropic second-order quadrupolar interactions that cannot be eliminated by MAS. ${ }^{[59]} \mathrm{New}$ composite pulses of the type $\left(\tau_{p}\right)_{x}\left(2 \tau_{p}\right)_{x}$ $\left(3 \tau_{p}\right)_{x}$ can achieve efficient excitation of the single-quantum coherences (SQC) associated with the central transitions and minimize the excitation of SQCs of satellite transitions and spurious coherences of higher order. Given that the $r$-manipulations are much shorter than in established approaches ( $\mu$ s instead of ms), our novel excitation schemes are referred to as 'Composite Pulses Adapted for Central Transitions' ('COMPACT'.) These methods have been tested successfully in both static and spinning solids containing spins with $I=3 / 2,5 / 2$ and $7 / 2$.

\section{Conclusions}

A unique feature of NMR in Lausanne is the cohabitation of both MAS-DNP and dissolution-DNP. The groups of Rolf Gruetter and Arnaud Comment develop complementary activities in the challenging field of in vivo DNP-MRI. The teams of Stefano Alberti and Jean-Philippe Ansermet are working on a new generation of gyrotrons. We have also developed collaborations with the Paul Scherrer Institute to exploit photo-excited triplet states for DNP and with the University of Geneva on polarized gases. Altogether, the EPFL has emerged as a unique centre of competence for boosting polarization in NMR and enjoys a world-wide reputation.

\section{Acknowledgements}

We are indebted to Jacques van der Klink, Arnaud Comment, J. A. (Ton) Konter, Patrick Hautle, Ben van den Brandt and Robero Melzi for numerous contributions to dissolution DNP instrumentation, and to Melanie Rosay, Frank Engelke and the staff of Bruker BioSpin for their help with solid-state MAS-DNP. This work was supported by the Swiss National Science Foundation (SNSF), the Commission pour la Technologie et l'Innovation (CTI), the Ecole Polytechnique Fédérale de Lausanne (EPFL), and the French CNRS.

Received: July 31, 2012
[1] A. Abragam, M. Goldman, Rep. Prog. Phys. 1978, 41,395

[2] J. H. Ardenkjaer-Larsen, B. Fridlund, A. Gram, G. Hansson, L. Hansson, M. H. Lerche, R. Servin, M. Thaning, K. Golman, Proc. Natl. Acad. Sci. USA 2003, 100, 10158

[3] A. Abragam, M. Goldman, 'Nuclear Magnetism: Order and Disorder', Int. Ser. Monographs. Phys., Clarendon Press, Oxford, 1982.

[4] D. G. Crabb, W. Meyer, Annu. Rev. Nucl. Part. Sci. 1997, 47, 67.

[5] S. Goertz, W. Meyer, G. Reicherz, Prog. Part. Nucl. Phys. 2002, 49, 403.

[6] K. N. Hu, G. T. Debelouchina, A. A. Smith, R. G. Griffin, J. Chem. Phys. 2011, 134.

[7] A. Abragam, W. G. Proctor, C.R. Hebd. Acad. Sci. 1958, 246, 2253.

[8] P. Mieville, PhD thesis, Ecole Polytechnique Fédérale de Lausanne (Lausanne), 2011

[9] C. S. Song, K. N. Hu, C. G. Joo, T. M. Swager, R. G. Griffin, J. Am. Chem. Soc. 2006, 128, 11385 .

[10] M. Goldman, Appl. Magn. Reson. 2008, 34, 219.

[11] S. Jannin, A. Comment, J. J. van der Klink, Appl. Magn. Reson. 2012, 43, 59.

[12] M. Goldman, 'Spin Temperature and Nuclear Magnetic Resonance in Solids', Oxford University Press, Oxford, 1970.

[13] R. A. Wind, M. J. Duijvestijn, C. Vanderlugt, A. Manenschijn, J. Vriend, Prog. Nucl. Magn. Reson. Spectrosc. 1985, 17, 33.

[14] A. B. Barnes, B. Corzilius, M. L. MakJurkauskas, L. B. Andreas, V. S. Bajaj, Y. Matsuki, M. L. Belenky, J. Lugtenburg, J. R. Sirigiri, R. J. Temkin, J. Herzfeld, R. G. Griffin, Phys. Chem. Chem. Phys. 2010, 12, 5861.

[15] V. Vitzthum, PhD thesis, Ecole Polytechnique Fédérale de Lausanne (Lausanne), 2012

[16] M. Rosay, L. Tometich, S. Pawsey, R. Bader, R. Schauwecker, M. Blank, P. M. Borchard, S. R. Cauffman, K. L. Felch, R. T. Weber, R. J. Temkin, R. G. Griffin, W. E. Maas, Phys. Chem. Chem. Phys. 2010, $12,5850$.

[17] R. G. Griffin, Nature 2010, 468, 381.

[18] A. Lesage, M. Lelli, D. Gajan, M. A. Caporini, V. Vitzthum, P. Mieville, J. Alauzun, A. Roussey, C. Thieuleux, A. Mehdi, G. Bodenhausen, C. Copéret, L. Emsley, J. Am. Chem. Soc. 2010, $132,15459$.

[19] M. Lelli, D. Gajan, A. Lesage, M. A. Caporini, V. Vitzthum, P. Mieville, F. Heroguel, F. Rascon, A. Roussey, C. Thieuleux, M. Boualleg, L. Veyre, G. Bodenhausen, C. Copéret, L. Emsley, J. Am. Chem. Soc. 2011, 133, 2104.

[20] V. Vitzthum, P. Mieville, D. Carnevale, M. A. Caporini, D. Gajan, C. Copéret, M. Lelli, A. Zagdoun, A. J. Rossini, A. Lesage, L. Emsley, G. Bodenhausen, Chem. Commun. 2011, 48, 1988.

[21] J. Haber, J. H. Block, B. Delmon, Pure Appl. Chem. 1995, 67, 1257.

[22] M. Pruski, D. P. Lang, C. Fernandez, J. P. Amoureux, Solid State Nucl. Magn. Reson. 1997, 7, 327 .

[23] A. Comment, B. van den Brandt, K. Uffmann, F. Kurdzesau, S. Jannin, J. A. Konter, P. Hautle, W. T. H. Wenckebach, R. Gruetter, J. J. van der Klink, Concept. Magn. Reson. B 2007, 31B, 255.

[24] A. Comment, S. Jannin, J. N. Hyacinthe, P. Mieville, R. Sarkar, P. Ahuja, P. R. Vasos, X. Montet, F. Lazeyras, J. P. Vallee, P. Hautle, J. A. Konter, B. van den Brandt, J. P. Ansermet, R. Gruetter, G. Bodenhausen, Phys. Rev. Lett. 2010, 105, 018104

[25] J. Kurhanewicz, D. B. Vigneron, K. Brindle, E. Y. Chekmenev, A. Comment, C. H. Cunningham, R. J. DeBerardinis, G. G. Green, M. O. Leach, S. S. Rajan, R. R. Rizi, B. D. 
Ross, W. S. Warren, C. R. Malloy, Neoplasia 2011, 13, 81 .

[26] R. B. van Heeswijk, K. Uffmann, A. Comment, F. Kurdzesau, C. Perazzolo, C. Cudalbu, S. Jannin, J. A. Konter, P. Hautle, B. van den Brandt, G. Navon, J. J. van der Klink, R. Gruetter, Magn. Reson. Med. 2009, 61, 1489.

[27] P. Mieville, S. Jannin, L. Helm, G. Bodenhausen, J. Am. Chem. Soc. 2010, 132, 5006.

[28] A. Bornet, S. Jannin, G. Bodenhausen, Chem. Phys. Lett. 2011, 512, 151.

[29] P. R. Vasos, A. Comment, R. Sarkar, P. Ahuja, S. Jannin, J. P. Ansermet, J. A. Konter, P. Hautle, B. van den Brandt, G. Bodenhausen, Proc. Natl. Acad. Sci. USA 2009, 106, 18469.

[30] P. Mieville, S. Jannin, G. Bodenhausen, $J$. Magn. Reson. 2011, 210, 137.

[31] P. Mieville, P. Ahuja, R. Sarkar, S. Jannin, P. R. Vasos, S. Gerber-Lemaire, M. Mishkovsky, A. Comment, R. Gruetter, O. Ouari, P. Tordo, G. Bodenhausen, Angew. Chem. Int. Ed. 2010, 49, 7834.

[32] J. H. Ardenkjaer-Larsen, A. M. Leach, N. Clarke, J. Urbahn, D. Anderson, T. W. Skloss, NMR Biomed. 2011, 24, 927.

[33] M. Batel, M. Krajewski, K. Weiss, O. With, A. Dapp, A. Hunkeler, M. Gimersky, K. P. Pruessmann, P. Boesiger, B. H. Meier, S Kozerke, M. Ernst, J. Magn. Reson. 2012, 214, 166.
[34] A. J. Perez Linde, PhD thesis, University of Notthingham (Nottingham), 2010.

[35] A. Bornet, R. Melzi, S. Jannin, G. Bodenhausen, Appl. Magn. Reson. 2012, 43, 107.

[36] S. Jannin, A. Bornet, S. Colombo, G. Bodenhausen, Chem. Phys. Lett. 2011, 517, 234.

[37] S. Jannin, A. Bornet, R. Melzi, G. Bodenhausen, Chem. Phys. Lett. 2012, http://dx.doi. org/10.1016/j.cplett.2012.08.017.

[38] J. A. Aguilar, M. Nilsson, G. Bodenhausen, G. A. Morris, Chem. Commun. 2012, 48, 811.

[39] B. Baishya, T. F. Segawa, G. Bodenhausen, $J$. Magn. Reson. 2011, 211, 240.

[40] D. Carnevale, T. F. Segawa, G. Bodenhausen, Chem. Eur. J. 2012, 18, 11573.

[41] N. Salvi, S. Ulzega, F. Ferrage, G. Bodenhausen, J. Am. Chem. Soc. 2012, 134, 2481.

[42] S. Ulzega, N. Salvi, T. F. Segawa, F. Ferrage, G. Bodenhausen, ChemPhysChem 2011, 12, 333.

[43] M. Verde, S. Ulzega, F. Ferrage, G. Bodenhausen, J. Chem. Phys. 2009, 130, 074506.

[44] M. H. Levitt, Annu. Rev. Phys. Chem. 2012, 63, 89.

[45] R. Sarkar, P. Ahuja, P. R. Vasos, G. Bodenhausen, Phys. Rev. Lett. 2010, 104, 053001.

[46] R. Sarkar, P. Ahuja, P. R. Vasos, A. Bornet, O. Wagnieres, G. Bodenhausen, Prog. Nucl. Magn. Reson. Spectrosc. 2011, 59, 83.
[47] N. Salvi, R. Buratto, A. Bornet, S. Ulzega, I. Rentero Rebollo, A. Angelini, C. Heinis, G. Bodenhausen, J. Am. Chem. Soc. 2012, 134, 11076

[48] A. Bornet, S. Jannin, J. A. Konter, P. Hautle, B. van den Brandt, G. Bodenhausen, J. Am. Chem. Soc. 2011, 133, 15644.

[49] S. Chinthalapalli, A. Bornet, T. F. Segawa, R. Sarkar, S. Jannin, G. Bodenhausen, Phys. Rev. Lett. 2012, 109, 047602.

[50] T. Giavani, H. Bildsoe, J. Skibsted, H. J. Jakobsen, J. Phys. Chem. B 2002, 106, 3026.

[51] S. Cavadini, A. Abraham, G. Bodenhausen, Chem. Phys. Lett. 2007, 445, 1.

[52] S. Cavadini, S. Antonijevic, A. Lupulescu, G. Bodenhausen, J. Magn. Reson. 2006, 182, 168

[53] S. Cavadini, S. Antonijevic, A. Lupulescu, G. Bodenhausen, ChemPhysChem 2007, 8, 1363.

[54] S. Ulzega, Chimia 2010, 64, 157.

[55] S. Cavadini, A. Abraham, S. Ulzega, G. Bodenhausen, J. Am. Chem. Soc. 2008, 130, 10850.

[56] G. A. Morris, R. Freeman, J. Magn. Reson. 1978, 29, 433.

[57] V. Vitzthum, M. A. Caporini, S. Ulzega, G. Bodenhausen, J. Magn. Reson. 2011, 212, 234.

[58] D. Carnevale, G. Bodenhausen, Chem. Phys. Lett. 2012, 530, 120.

[59] M. J. Duer, 'Solid-State NMR Spectroscopy: Principles and Applications', Blackwell Science, UK, 2002. 